\title{
Role for artificial intelligence in respiratory diseases-chronic obstructive pulmonary disease
}

\author{
Chris Carlin $^{1}$, Anna Taylor ${ }^{1}$ Isabel van Loon ${ }^{1}$, Grace McDowell ${ }^{1}$, Shane Burns ${ }^{2}$, Paul McGinness ${ }^{2}$, \\ David J. Lowe ${ }^{3}$
}

${ }^{1}$ Department of Respiratory Medicine, Queen Elizabeth University Hospital, Glasgow, Scotland, UK; ${ }^{2}$ StormID, Leith Assembly Rooms, Edinburgh, Scotland, UK; ${ }^{3}$ Department of Emergency Medicine, Queen Elizabeth University Hospital, Glasgow, Scotland, UK

Contributions: (I) Conception and design: C Carlin, P McGinness, DJ Lowe; (II) Administrative support: All authors; (III) Provision of study material or patients: None; (IV) Collection and assembly of data: All authors; (V) Data analysis and interpretation: All authors; (VI) Manuscript writing: All authors; (VII) Final approval of manuscript: All authors.

Correspondence to: Dr. Chris Carlin. Department of Respiratory Medicine, Queen Elizabeth University Hospital, Glasgow G51 4TF, UK.

Email: christopher.carlin@ggc.scot.nhs.uk.

\begin{abstract}
Chronic obstructive pulmonary disease (COPD) is a global healthcare challenge. It is highly prevalent in low-income countries, causes 3 million death per year and is projected to be the leading cause of death globally by 2030. Challenges in COPD management result in care quality gaps which impair timely and accurate diagnosis and limit patient stratification and provision of evidence-based interventions. COPD exacerbations are responsible for a large proportion of the disease-burden, adverse outcomes and healthcare costs. There is a requirement to re-orientate COPD exacerbation care from failure-driven reactive approach to one based on proactive preventative management. Service model adaptation supported by artificial intelligence (AI) tools offer the prospect of addressing these care-quality gaps and achieving this practice re-orientation. Progress with clinical applications of AI for COPD is accelerating. Evidence available demonstrates the potential of AI techniques to facilitate early and precise COPD case-finding and diagnosis, allow stratification with clinical decision support to prioritise management, and achieve accurate exacerbation detection/prediction to allow proactive interventions. In this narrative review, we will summarise current evidence for the application of AI to these COPD challenges, outline the barriers to implementation of AI models and present our opinion on the required next steps to realise the potential role for AI in COPD management.
\end{abstract}

Keywords: Artificial intelligence (AI); chronic obstructive pulmonary disease (COPD); machine-learning

Received: 25 August 2020; Accepted: 28 January 2021; Published: 25 September 2021.

doi: $10.21037 /$ jhmhp-20-119

View this article at: http://dx.doi.org/10.21037/jhmhp-20-119

\section{Introduction}

Chronic obstructive pulmonary disease (COPD) is a common, progressive, preventable and treatable respiratory disorder. It arises in susceptible patients as a result of environmental exposures, principally tobacco smoking and indoor air pollution. Early life events including those which impair lung development contribute to COPD susceptibility and pathogenesis (1). COPD affects an estimated 328 million people worldwide, is the $2^{\text {nd }}$ commonest reason for emergency hospital admissions, and is projected to be the leading cause of death globally by 2030 (2). COPD patients experience progressive symptoms, accelerated lung function decline and an impaired quality of life (3). Exacerbations of

^ ORCID: 0000-0002-8520-7224. 
COPD—where there are acute deteriorations in symptoms which have a variety of triggers including infections-are major life events. COPD exacerbations are responsible for a large part of the individual disease burden, and adverse outcomes of hospital admission, readmission and death. Exacerbations are responsible for a large part of the direct healthcare and indirect economic costs of COPD (4-6).

The NHS commissioned Topol Report noted that advances in mathematics, computing power, cloud computing and algorithm design have accelerated the development of artificial intelligence (AI) based methods to analyse, interpret and make predictions based on healthcare data (7). There has been an associated surge in interest in these clinical applications of $\mathrm{AI}$ including in the respiratory medicine field, where there has been a substantial increase in publications since 2016 (8). Applications of AI directed at the global COPD challenge offers the potential to address the noted priority requirements of earlier and accurate diagnosis and personalised, predictive, preventative management (9). COPD has been identified as an ideal field to establish clinical, academic and industrial partnerships which resolve variations in care with the development of exemplar AI solutions ranging from imaging and lung function test interpretation to predictive applications.

For example, there has been considerable progress in our understanding and recognition of COPD at its presymptomatic stages. AI-based evaluation of chest CT imaging could identify COPD, and provide call to action which would allow interventions to slow or prevent disease progression (10), Heterogeneity of COPD is also notable, with an emerging role for stratification of acute and long term COPD treatment based on biomarkers (3). AI tools could integrate the source data and provide decision support to ensure optimised care, rationalising specialist clinician workload and reducing variations in care. COPD use cases have the potential to serve as exemplars for clinical validation of $\mathrm{AI}$ applications, and to be used as accelerators to address some of the generic challenges for AI implementation including interoperability, interpretability, data governance and patient and clinician readiness.

The COVID-19 pandemic presents additional challenges but also opportunities for clinical AI in general, and for its application in COPD management. COPD patients have an increased risk of severe disease and mortality from COVID-19, which amplifies the potential individual and healthcare system burden (11). COVID-19 society lockdowns and patient-carer shielding requirements have interrupted routine healthcare, particularly diagnostic and monitoring lung function testing and pulmonary rehabilitation which conventionally requires group face-face care sessions. The COVID-19 challenge is accelerating adoption of digital technologies within the general population, and of remote-management and associated digital innovations to re-orientate and continue scheduled healthcare $(12,13)$. It seems not just appropriate but necessary to seize the opportunity to 'piggyback' AI validation and implementation on COVID-19 response workstreams. This offers the potential to maximise the gains from the source data now obtained from enhanced remote-monitoring and care model paradigm shifts, and from the effort being undertaken to accelerate resolution of the connectivity, interoperability, governance and training requirements for these re-orientated care systems.

In this review, we will outline the background and current state of AI applications for COPD diagnosis, stratification, outcome detection and prediction and present our vision for the role in the immediate future for $\mathrm{AI}$ in COPD, including the validation and implementation requirements.

\section{Current state: Al and COPD diagnosis}

COPD diagnosis is traditionally based on presence of symptoms and risk factors (typically smoking or home air pollution) and results of lung function testing. Spirometry testing demonstrating fixed airflow obstruction-ratio of the forced expiratory volume in 1 second (FEV1) to the forced vital capacity (FVC) $<0.7$-in the appropriate clinical context establishes the diagnosis of COPD. COPD is then classified and graded by symptom burden, exacerbation frequency and the severity of lung function impairment based on the FEV1 measured vs predicted. Whilst this disease definition and classification informs prognosis and treatment stratification, several problems including under and over diagnosis and over-simplification of disease heterogeneity are noted (14). Performance and interpretation of spirometry requires access to calibrated equipment and time for test conduct. Accurate interpretation requires clinical expertise, with normal ranges based on reference datasets which have limitations including age and ethnicity biases (15). AI applications have the potential to address these shortfalls by optimising data interpretation for population based factors, to derive maximal value and accuracy from traditional lung function testing. AI algorithms also have the potential to extend the reach of other diagnostic modalities, to match these with clinical outcomes which should equate to a COPD diagnosis 
and treatment provision.

The use of early rule-based expert system AI techniques to interpret pulmonary function test was originally explored in the 1980s. The model outputs weren't robust, with computing power at that time limiting their development and utility (16). If, however we fast forward 30 years, modern AI has the ability to find patterns in high dimensional feature space and apply these to PFT interpretation. For example, Topalovic et al. developed a ML framework that incorporates computer-based choice of lung function and clinical features to enhance diagnosis of COPD and other common respiratory conditions from PFT measurements (17). In subsequent validation study performed on 50 cases, this AI model outperformed clinician diagnosis, with an accuracy of $82 \%$ for the model vs. $44 \%$ with large inter-rater variability for the clinicians (18). At face value AI interpretation of lung function testing is ready for adoption at scale. The challenge is to define the service model within which it would fit: as a trigger to refer for expert review within a community-based lung function service, or as an interpretable and responsible AI model 'leading' on lung function test reporting, with intermittent expert clinician calibration and model tuning? An expansion of the lung function AI training dataset to reflect the global population, address varying disease prevalence in cohorts, and remove biases is required. Data from international COPD projects could be exploited for this (19). Digitally connected handheld spirometers with patient's coached through full forced or a more limited and repeatable set of slow breathing manoeuvres have been validated for testing including community screening for COPD (20-22). Innovative approaches to measure standard pulmonary function variables using smartphone microphone are in advanced development (23). COVID-19 challenges are accelerating the uptake of these solutions (24). Further trials are proposed to evaluate utility of service models supported by connected spirometry vs standard service models. Datasets from these are in cloud storage, and could be accessed to train and validate algorithms for measurement quality and variability, diagnosis and stratification. The hope would be to aggregate spirometry datasets with patient-reported outcome, biosensor, imaging and supervised clinical event data to enhance algorithm development for detection or prediction of exacerbations and other outcomes. Even without these attractive additions, AI applications will be necessary to address the overwhelming data quantity from connected lung function, to ensure comprehensive utilisation and provide actionable decision support to clinical teams.

Lung function testing also can highlight subtler abnormalities which are not typically noted or actioned in routine clinical practice. These insights can predict subsequent development of COPD, with adverse outcomes (25). Surfacing these insights is another approach by which AI algorithm development can potentially maximise the return from lung function testing, contributing to accurate and timely diagnosis and preventative management strategy.

Detection and classification of imaging abnormalities has been one of the headline applications of clinical AI. Access to CT scanners, expanded indications for 'routine' imaging and progression of imaging technology including increased resolution and standardised picture archiving and storage have underpinned this.

Initial ML techniques applied to the COPD diagnosis challenge employed textural analysis of CT scans, where features were extracted by applying algorithms based on structure, statistics or binary patterns to discrete regions of interest within the lung field (26). The derived model was able to identify obstructive patterns within lung parenchyma and address issues with airway segmentation demonstrating proof of concept, but this ML approach requires significant supervision and lacks interoperability which limits its scaleability. The development and application of deep learning AI techniques, in particular convolutional neural networks (CNN) has largely overcome these issues. Deep-learning model training and validation however requires large deidentified data sets with reference labelling, intensive computer resource and an infrastructure for subsequent model implementation. These requirements are being addressed by innovation hubs such as iCAIRD (27). Caution is required with model training and supervision to mitigate overfitting and convergence issues (16). Language processing AI techniques have recently been successfully applied to imaging reports, showing potential to reduce supervision requirements and accelerate derivation of accurate ground truth, mitigating these risks (28). As an example of this most current AI applications to COPD diagnosis, Gonzales et al have shown how CNN can accurately categorize CT images from smokers, differentiating presence or absence of COPD (29). A CNN model an also accurately classify severity of COPD based on GOLD stage, with the ability to detect mild COPD (30). The recently described 'SuStain' ML model when applied to CT thorax images from asymptomatic smokers who have been followed up can identify subgroup of $30 \%$ of patients who have a $2.5 \mathrm{x}$ relative risk of developing COPD 
subsequently (31). These findings highlight the potential of AI algorithms to accurately establish early diagnosis of COPD at scale, enabling preventative interventions. CT images of the thorax are widely obtained in patients at risk for COPD in emergency (chest pain, trauma) and screening (lung cancer) settings, and clinically acceptable performance of an AI model for COPD diagnosis from these scans has been confirmed (32). The challenge now is to implement the AI models from imaging, lung function and other sources, and establish the effectiveness of a service model associated with these that can interpret and action the enhanced diagnostic information that can be derived from repurposing of this existing healthcare data. From this, it is anticipated that a new diagnostic paradigm for COPD will emerge, supported by validated comprehensive AI evaluation of healthcare data which associates with outcomes and personalises management (33).

\section{Current state: AI and COPD stratification}

Whilst traditionally regarded as a single diagnostic entity, with diagnosis still based on threshold criteria (age, symptoms, exposures, airflow obstruction on spirometry) COPD is better considered a syndrome, with a variety of different phenotypes. Basic phenotyping of COPD by airflow obstruction severity, symptom burden and exacerbation history is well established, and it determines patient cohorts with different outcomes and different primary treatment strategies (3). The evidence-base for range of variables which stratify COPD outcomes and treatment strategies has expanded rapidly. Laboratory results (serum CRP and eosinophil count) have been shown to identify patients with COPD exacerbations who can have antibiotics withheld or oral corticosteroid course truncated $(34,35)$. Absence of exacerbations, low blood eosinophil count, sputum bacterial isolates, previous pneumonia episodes and occurrence of oral thrush differentiate COPD patients who should potentially have inhaled corticosteroids omitted or withdrawn from their treatment package (3). Acute hypercapnic respiratory failure is an indication for acute non-invasive ventilation; sustained hypoxaemia in ex-smokers indicates home oxygen therapy (36); patients with sustained hypercapnic respiratory failure benefit from home NIV (37); patients with hyperinflation, persisting exercise impairment after pulmonary rehabilitation, low lung infection risk, stable comorbidities and favourable pulmonary anatomy benefit from lung volume reduction procedures (38). COPD commonly co-exists or overlaps with asthma, cardiovascular disease, obesity, obstructive sleep apnoea, spinal degenerative disease, cachexia and lung cancer. Each of these combinations result in mutually unfavourable aggravated symptom-burden and outcomes. COPD exacerbations are a major life event with high personal and societal cost. Risk prediction scores such as DECAF (39), or newer physiology techniques measuring expiratory flow limitation (40) or neural respiratory drive index (41) can highlight patients with COPD exacerbations at higher risk for early re-admission or other adverse outcomes. Evaluation of these stratifying variables and detection and management of these comorbidities is recommended to optimise COPD management.

It is unclear whether combining some or all of these established COPD stratifying variables, or integrating them with other accessible clinical data improves the precision of phenotyping, allowing stratification of patients to prioritised personalised interventions.

The complexity of this matrix of potential COPD stratifications exceeds cognitive capacity for even an expert clinician at a single care episode. Despite intensive quality improvement work including attempts to develop and implement COPD care bundles, providing decision support across one axis of care (acute and early post exacerbation management without consideration of comorbidities), considerable variations in care continue to exist even for the apparently simplest binary management points $(42,43)$. Multi-disciplinary follow up care of COPD is recommended, but this is expensive and global provision is unrealistic.

$\mathrm{AI}$ applications offer the tantalising potential to address these challenges. If we can integrate the above evidencebased data points and situational variables in a secure, consent-driven data exchange and input these to validated models at scale, there is on the immediate horizon the potential to provide COPD patients and clinicians with prioritised and explainable decision support.

Research reported over the last 3 years suggests that this vision for AI-based COPD stratification can be realised. Most investigators have focused on exacerbation, admission/ re-admission or mortality predictions, which is appropriate as these are priority outcomes for patients and providers. The identification of high risk of readmission or mortality has potential for immediate change in a patient's care: intensified support and anticipatory care planning. Guerra and colleagues noted in 2017 that despite 25 publications reporting on COPD exacerbation prediction and riskstratification models, methodology had been inconsistent, 
no published model met clinical requirements and that a more harmonised approach to model development and validation was required (44). That challenge has been partly addressed, with several subsequent publications demonstrating that machine-learning techniques can predict in-hospital mortality and 30-day readmission risk for COPD exacerbations, differentiate high-cost COPD patients (with cost as a proxy for hospital admissions), predict likelihood of hospital admission from emergency department triage and predict emergency care utilisation based on weather and environmental variables (45-51). These approaches have scale-ability hurdles, requiring data consistency and need for validation +/- re-training on other population cohorts. Use of a smooth Bayesian network ML technique can potentially mitigate data sparsity issues, and also provide quantitative relationships which enhances model explainability (52). Pikoula and colleagues have also shown that it is feasible to obtain clinically important COPD stratification from core primary care electronic health record data, which offers prospect to reduce the requirement for model data input and expert clinical reviews (53).

Most recently, investigators have shown impressive performance of an ML model for all-cause mortality, superior to previous validated mortality risk scores, using training and validation data from the large COPD gene epidemiology and ECLIPSE COPD longitudinal study datasets (54). This machine-learning mortality prediction (MLMP-COPD) model can be accessed online (55). The results of this study are encouraging, but not immediately scale-able: the feature set for the MLMP-COPD model was clinician selected rather than data-driven, and few of the model input features are routinely acquired and recorded in real world practice. Stratification models which are based on more widely accessible consumer health wearable data are emerging (56), and combing these with the academic approaches which have proven valid for COPD use cases is an obvious next step.

\section{Current state: AI and COPD outcome detection and prediction}

Reducing COPD exacerbations and hospitalisations is a global health priority. AI is likely to have a central role tackling this challenge.

Systematic review by Zhang and colleagues has established that exacerbations and hospitalisations due to exacerbations are the outcomes that COPD patients rate as most important (57). Management strategies focusing on detecting and preventing exacerbations in the community are likely to improve individual outcomes and quality of life, and reduce COPD healthcare and society costs (58). In addition to the noted background health and situational data which can predict risk of hospitalisation and re-admission, there is a spectrum of data which has the potential to detect and predict COPD exacerbations. Patient-reported outcomes (PROs) vary with exacerbations, with changes typically occurring days prior to healthcare contact (59). The feasibility of capturing these PROs via smartphone app, and tracking changes which equate to exacerbations has been confirmed (60). Changes in symptom relieving short-acting bronchodilator inhaler use (61), and in patients with severe disease changes in home non-invasive ventilation parameters occur at early stages of COPD exacerbations $(62,63)$. Heart rate and activity changes from wearable devices can follow trends of respiratory infections at a population level and potentially detect developing illness and need for hospitalisation $(64,65)$. As of 2016, systematic review noted that there was promise but insufficient data on monitoring of physiology parameters (such as heart rate, activity, respiratory rate or oxygen saturation) for remote-monitoring and exacerbation prediction at an individual patient level (66). That promise is being realised: ML models outperform other approaches for exacerbation detection and prediction from datasets of PROs and physiology data acquired from digital COPD remote-monitoring interfaces $(67,68)$. These are proofs of concept: larger datasets with higher volume of events are required for further model training and validation.

The rapid expansion of access and uptake of smartphone and wearable technology provides the opportunity to build on these proofs of concept with much larger datasets. Expanding patient ownership of smartphones and acceptance of health monitoring and digital selfmanagement of COPD is notable $(69,70)$. COVID-19 challenges are further accelerating digital connectivity and the uptake of continuous remote-monitoring for health. Processing and cleaning of this data will be facilitated by cloud computing, but clinical supervision is required and consistent methodology and reporting of remotemonitoring solutions still needs attention (71). That clinical supervision importantly includes accurately defining exacerbations (a generic priority for the COPD academic clinical community) and timings to provide ground truth for model training. Based on these developments, the hope is that $\mathrm{AI}$ algorithms will have a role at an individual patient level, predicting adverse outcome such as exacerbation 
and providing decision support to re-orientate care within an end-end remote-monitoring service model. Evidence for this needs more time to accrue, and implementation and evaluation challenges to scale this are notable. In the immediate future, AI models which are trained and validated on comprehensive remote monitoring datasets will provide key insights into feature set selection. This should allow us to prioritise data sources for remotemonitoring, such as defining which physiology parameters and associated hardware are most valuable for measurement and data integration. The step beyond this-AI models demonstrating need for prioritised patient review and escalation of care based on remote-monitoring variables with explainable actionable insights-would naturally follow.

\section{Role for AI in improving COPD outcomes worldwide: immediate challenges and next steps}

AI applications hold great promise to improve healthcare outcomes. COPD is an ideal use case for AI solutions given the global burden of the disease, the inequalities and carequality gaps and its responsibility for a high proportion of healthcare spend. Current evidence base confirms the potential of a suite of AI applications and models to address these issues and demonstrate return on investments with accurate and timely COPD diagnosis, stratification to realise precision medicine and risk predictions to reorientate to proactive preventative care.

There are immediate challenges and significant barriers to implementing AI solutions at scale. These need to be addressed if positive research findings obtained are to be exploited with extension of the COPD-AI evidence-base and conduct of intervention trials with cost-effectiveness evaluations. The required data to train, validate and monitor AI model performance is in silos, and data control requirements are challenging to overcome $(72,73)$. Enforcement of GDPR legislation in the EU presents challenges around how patient-generated data can be used to support service delivery and research. Medical device regulatory review and approval of $\mathrm{AI}$ solutions requires clinical effectiveness evaluations. AI model outputs in isolation don't re-orientate care: an end-end digitallyenabled service model with clinician expertise to interpret and action these is required (7).

COPD services are well suited to address these challenges. Academic, commercial and healthcare organisation collaborations-the triple helix-are well established in the COPD field. Connected care with remote-patient monitoring has been pioneered in COPD management, with notable successes (74). These COPD multi-disciplinary teams are well placed to utilise existing skills and adapt systems and processes to evaluate AI solutions. Required infrastructure elements for AI delivery interoperability of health systems and data sources based on open standards, data storage aggregating patient-generated and background electronic health record data, consentassurance with privacy integrated into the architecture and de-identified data pipelines with APIs allowing real time analyses - are being evaluated in COPD clinical trials (75).

Based on all the positive data now reported, we believe that the next step for establishing a role for AI in COPD is to undertake a programme of implementation-effectiveness evaluations. Exemplar projects should establish clinical innovation test beds with collaborations, infrastructure, end-end digitally enabled service models and an evaluation framework capturing agreed health technology evaluation metrics, exploiting the analytic data from the digital infrastructure to semi-automate effectiveness testing. Within these test beds AI models can be rapidly developed, trained, validated and performance monitored, with associated regulatory oversight and an established pathway to scale-up.

The approaches required to tackle the COPD global healthcare challenge can be leveraged to address healthcare AI development needs. In turn, healthcare AI solution's capabilities can be leveraged to address the challenges of COPD global care. COPD's role as a priority use case for $\mathrm{AI}$ development is apparent, and a role for AI in COPD management is established. We expect the breadth and depth of that role to continue to expand. If healthcare AI's promise can be confirmed and implemented at scale, improvements in COPD outcomes at an individual and population level will be realised.

\section{Acknowledgments}

Funding: None.

\section{Footnote}

Provenance and Peer Review: This article was commissioned by the Guest Editors (Sandeep Reddy, Jenifer Sunrise Winter, and Sandosh Padmanabhan) for the series "AI in Healthcare - Opportunities and Challenges" published in Fournal of Hospital Management and Health Policy. The article 
has undergone external peer review.

Conflicts of Interest: All authors have completed the ICMJE uniform disclosure form (available at http://dx.doi. org/10.21037/jhmhp-20-119). The series “AI in Healthcare - Opportunities and Challenges" was commissioned by the editorial office without any funding or sponsorship. All authors report that NHS Greater Glasgow \& Clyde have a joint working agreement with StormID for the development and implementation of AI models to optimise COPD care. The authors have no other conflicts of interest to declare.

Ethical Statement: The authors are accountable for all aspects of the work in ensuring that questions related to the accuracy or integrity of any part of the work are appropriately investigated and resolved.

Open Access Statement: This is an Open Access article distributed in accordance with the Creative Commons Attribution-NonCommercial-NoDerivs 4.0 International License (CC BY-NC-ND 4.0), which permits the noncommercial replication and distribution of the article with the strict proviso that no changes or edits are made and the original work is properly cited (including links to both the formal publication through the relevant DOI and the license). See: https://creativecommons.org/licenses/by-nc-nd/4.0/.

\section{References}

1. Agustí A, Hogg JC. Update on the Pathogenesis of Chronic Obstructive Pulmonary Disease. N Engl J Med 2019;381:1248-56.

2. Quaderi SA, Hurst JR. The unmet global burden of COPD. Glob Health Epidemiol Genom 2018;3:e4.

3. Gold Reports for Personal Use. Global Initiative for Chronic Obstructive Lung Disease - GOLD. [cited 2020 Aug 16]. Available online: https://goldcopd.org/goldreports/

4. Wedzicha JA, Wilkinson T. Impact of chronic obstructive pulmonary disease exacerbations on patients and payers. Proc Am Thorac Soc 2006;3:218-21.

5. Ford ES, Murphy LB, Khavjou O, et al. Total and StateSpecific Medical and Absenteeism Costs of COPD Among Adults Aged 18 Years in the United States for 2010 and Projections Through 2020. CHEST 2015;147:31-45.

6. McLean S, Hoogendoorn M, Hoogenveen RT, et al. Projecting the COPD population and costs in England and Scotland: 2011 to 2030. Sci Rep 2016;6:31893.
7. The Topol Review - NHS Health Education England. The Topol Review - NHS Health Education England. [cited 2020 Aug 16]. Available online: https://topol.hee. nhs.uk/

8. Gonem S, Janssens W, Das N, et al. Applications of artificial intelligence and machine learning in respiratory medicine. Thorax 2020;75:695-701.

9. Agusti A, Alcazar B, Cosio B, et al. Time for a change: anticipating the diagnosis and treatment of COPD. Eur Respir J 2020;56:2002104.

10. Labaki WW, Martinez CH, Martinez FJ, et al. The Role of Chest Computed Tomography in the Evaluation and Management of the Patient with Chronic Obstructive Pulmonary Disease. Am J Respir Crit Care Med 2017;196:1372-9.

11. Pharmatics. Meta-analysis of severity and mortality of COVID-19 in COPD [Internet]. 2020. Available online: https://med-ai.uk/covid

12. Online Nation: narrative report. Ofcom. 2020 [cited 2020 Aug 24]. Available online: https://www.ofcom.org.uk/ research-and-data/internet-and-on-demand-research/ online-nation/narrative

13. Mosnaim GS, Stempel H, Van Sickle D, et al. The Adoption and Implementation of Digital Health Care in the Post-COVID-19 Era. J Allergy Clin Immunol Pract. 2020 Jun 22 [cited 2020 Aug 17]. Available online: http://www.sciencedirect.com/science/article/pii/ S2213219820306048

14. Diab N, Gershon AS, Sin DD, et al. Underdiagnosis and Overdiagnosis of Chronic Obstructive Pulmonary Disease. Am J Respir Crit Care Med 2018;198:1130-9.

15. Stanojevic S, Wade A, Stocks J. Reference values for lung function: past, present and future. Eur Respir J 2010;36:12-9.

16. Das N, Topalovic M, Janssens W. Artificial intelligence in diagnosis of obstructive lung disease: current status and future potential. Curr Opin Pulm Med 2018;24:117-23.

17. Topalovic M, Laval S, Aerts JM, et al. Automated Interpretation of Pulmonary Function Tests in Adults with Respiratory Complaints. Respiration 2017;93:170-8.

18. Topalovic M, Das N, Burgel P-R, et al. Artificial intelligence outperforms pulmonologists in the interpretation of pulmonary function tests. Eur Respir J 2019;53:1801660.

19. Siddharthan T, Pollard SL, Quaderi SA, et al. Effectiveness-implementation of COPD case finding and self-management action plans in low- and middle-income countries: global excellence in COPD outcomes (GECo) 
study protocol. Trials 2018;19:571.

20. Masa JF, González MT, Pereira R, et al. Validity of spirometry performed online. Eur Respir J 2011;37:911-8.

21. Exarchos KP, Gogali A, Sioutkou A, et al. Validation of the portable Bluetooth ${ }^{\circledR}$ Air Next spirometer in patients with different respiratory diseases. Respir Res 2020;21:79.

22. Dickens AP, Fitzmaurice DA, Adab P, et al. Accuracy of Vitalograph lung monitor as a screening test for COPD in primary care. Npj Prim Care Respir Med 2020;30:2.

23. Chung H, Jeong C, Luhach AK, et al. Remote Pulmonary Function Test Monitoring in Cloud Platform via Smartphone Built-in Microphone. Evol Bioinform Online 2019;15:1176934319888904.

24. NHS England. Thousands of patients to benefit from NHS At Home roll-out. [cited 2020 Aug 16]. Available online: https://www.england.nhs.uk/2020/06/thousandsof-patients-to-benefit-from-nhs-at-home-roll-out/

25. Zeng S, Tham A, Bos B, et al. Lung volume indices predict morbidity in smokers with preserved spirometry. Thorax 2019;74:114-24.

26. Mining textural knowledge in biological images: Applications, methods and trends. [cited 2020 Aug 16]. Available online: https://www.ncbi.nlm.nih.gov/pmc/ articles/PMC5155047/

27. Home. iCAIRD. [cited 2020 Aug 16]. Available online: https://icaird.com/

28. Drozdov I, Forbes D, Szubert B, et al. Supervised and unsupervised language modelling in Chest X-Ray radiological reports. PLoS One 2020;15:e0229963.

29. González G, Ash SY, Vegas-Sánchez-Ferrero G, et al. Disease Staging and Prognosis in Smokers Using Deep Learning in Chest Computed Tomography. Am J Respir Crit Care Med 2018;197:193-203.

30. Fischer AM, Varga-Szemes A, Martin SS, et al. Artificial Intelligence-based Fully Automated Per Lobe Segmentation and Emphysema-quantification Based on Chest Computed Tomography Compared With Global Initiative for Chronic Obstructive Lung Disease Severity of Smokers. J Thorac Imaging 2020;35 Suppl 1:S28-34.

31. Young AL, Bragman FJS, Rangelov B, et al. Disease Progression Modeling in Chronic Obstructive Pulmonary Disease. Am J Respir Crit Care Med 2020;201:294-302.

32. Tang LYW, Coxson HO, Lam S, et al. Towards large-scale case-finding: training and validation of residual networks for detection of chronic obstructive pulmonary disease using low-dose CT. Lancet Digit Health 2020;2:e259-67.

33. Celli BR, Agustí A. COPD: time to improve its taxonomy? ERJ Open Res 2018;4:00132-2017.
34. Butler CC, Gillespie D, White P, et al. C-Reactive Protein Testing to Guide Antibiotic Prescribing for COPD Exacerbations. N Engl J Med 2019;381:111-20.

35. Sivapalan P, Lapperre TS, Janner J, et al. Eosinophilguided corticosteroid therapy in patients admitted to hospital with COPD exacerbation (CORTICO-COP): a multicentre, randomised, controlled, open-label, noninferiority trial. Lancet Respir Med 2019;7:699-709.

36. Elliott MW, Nava S. Noninvasive Ventilation for Acute Exacerbations of Chronic Obstructive Pulmonary Disease: "Don't Think Twice, It's Alright!" Am J Respir Crit Care Med 2012;185:121-3.

37. Murphy PB, Rehal S, Arbane G, et al. Effect of Home Noninvasive Ventilation With Oxygen Therapy vs Oxygen Therapy Alone on Hospital Readmission or Death After an Acute COPD Exacerbation: A Randomized Clinical Trial. JAMA 2017;317:2177-86.

38. Gordon M, Duffy S, Criner GJ. Lung volume reduction surgery or bronchoscopic lung volume reduction: is there an algorithm for allocation? J Thorac Dis 2018;10:S2816-23.

39. Echevarria C, Steer J, Heslop-Marshall K, et al. Validation of the DECAF score to predict hospital mortality in acute exacerbations of COPD. Thorax 2016;71:133-40.

40. Aarli BB, Calverley PM, Jensen RL, et al. The association of tidal EFL with exercise performance, exacerbations, and death in COPD. Int J Chron Obstruct Pulmon Dis 2017;12:2179-88.

41. Suh E-S, Mandal S, Harding R, et al. Neural respiratory drive predicts clinical deterioration and safe discharge in exacerbations of COPD. Thorax 2015;70:1123-30.

42. Hurst JR, Legg M, Shanahan S, et al. National Asthma and Chronic Obstructive Pulmonary Disease Audit Programme (NACAP). COPD clinical audit 2018/19 (people with COPD exacerbations discharged from acute hospitals in England, Scotland and Wales between September 2018 and 2019). Clinical audit report. RCP. 2020 Jul.

43. Morton K, Sanderson E, Dixon P et al. Care bundles to reduce re-admissions for patients with chronic obstructive pulmonary disease: a mixed-methods study. Southampton (UK): NIHR Journals Library; 2019 Jun.

44. Guerra B, Gaveikaite V, Bianchi C, et al. Prediction models for exacerbations in patients with COPD. Eur Respir Rev 2017;26:160061.

45. Jain SS, Sarkar IN, Stey PC, et al. Using Demographic Factors and Comorbidities to Develop a Predictive Model for ICU Mortality in Patients with Acute Exacerbation COPD. AMIA Annu Symp Proc AMIA Symp 
2018;2018:1319-28.

46. Min X, Yu B, Wang F. Predictive Modeling of the Hospital Readmission Risk from Patients' Claims Data Using Machine Learning: A Case Study on COPD. Sci Rep 2019;9:2362.

47. Zhou M, Chen C, Peng J, et al. Fast Prediction of Deterioration and Death Risk in Patients With Acute Exacerbation of Chronic Obstructive Pulmonary Disease Using Vital Signs and Admission History: Retrospective Cohort Study. JMIR Med Inform 2019;7:e13085.

48. Peng J, Chen C, Zhou M, et al. Peak Outpatient and Emergency Department Visit Forecasting for Patients With Chronic Respiratory Diseases Using Machine Learning Methods: Retrospective Cohort Study. JMIR Med Inform 2020;8:e13075.

49. Khatri KL, Tamil LS. Early Detection of Peak Demand Days of Chronic Respiratory Diseases Emergency Department Visits Using Artificial Neural Networks. IEEE J Biomed Health Inform 2018;22:285-90.

50. Eckert C, Ahmad M, Zolfaghar K, et al. S45 Predicting likelihood of emergency department admission prior to triage: utilising machine learning within a COPD cohort. Thorax 2018;73:A28.

51. Goto T, Jo T, Matsui H, et al. Machine LearningBased Prediction Models for 30-Day Readmission after Hospitalization for Chronic Obstructive Pulmonary Disease. COPD 2019;16:338-43.

52. Sprooten RTM, Rohde GGU, Lawyer G, et al. Risk stratification for short-term mortality at hospital admission for acute exacerbations of COPD. Respirology 2019;24:765-76.

53. Pikoula M, Quint JK, Nissen F, et al. Identifying clinically important COPD sub-types using data-driven approaches in primary care population based electronic health records. BMC Med Inform Decis Mak 2019;19:86.

54. Moll M, Qiao D, Regan EA, et al. Machine Learning and Prediction of All-Cause Mortality in COPD. Chest 2020;158:952-64.

55. Available online: https://cdnm.shinyapps.io/ cgmortalityapp/

56. Strain T, Wijndaele K, Dempsey PC, et al. Wearabledevice-measured physical activity and future health risk. Nat Med 2020;26:1385-91.

57. Zhang Y, Morgan RL, Alonso-Coello P, et al. A systematic review of how patients value COPD outcomes. Eur Respir J 2018;52:1800222.

58. Punekar YS, Shukla A, Müllerova H. COPD management costs according to the frequency of COPD exacerbations in UK primary care. Int J Chron Obstruct Pulmon Dis 2014;9:65-73.

59. Mackay AJ, Kostikas K, Murray L, et al. Patient-reported Outcomes for the Detection, Quantification, and Evaluation of Chronic Obstructive Pulmonary Disease Exacerbations. Am J Respir Crit Care Med 2018;198:730-8.

60. Rodriguez Hermosa JL, Fuster Gomila A, Puente Maestu L, et al. Compliance and Utility of a Smartphone App for the Detection of Exacerbations in Patients With Chronic Obstructive Pulmonary Disease: Cohort Study. JMIR MHealth UHealth 2020;8:e15699.

61. Sumino K, Locke ER, Magzamen S, et al. Use of a Remote Inhaler Monitoring Device to Measure Change in Inhaler Use with Chronic Obstructive Pulmonary Disease Exacerbations. J Aerosol Med Pulm Drug Deliv 2018;31:191-8.

62. Borel JC, Pelletier J, Taleux N, et al. Parameters recorded by software of non-invasive ventilators predict COPD exacerbation: a proof-of-concept study. Thorax 2015;70:284-5.

63. Blouet S, Sutter J, Fresnel E, et al. Prediction of severe acute exacerbation using changes in breathing pattern of COPD patients on home noninvasive ventilation. Int J Chron Obstruct Pulmon Dis 2018;13:2577-86.

64. Radin JM, Wineinger NE, Topol EJ, et al. Harnessing wearable device data to improve state-level real-time surveillance of influenza-like illness in the USA: a population-based study. Lancet Digit Health 2020;2:e85-93.

65. Natarajan A, Su HW, Heneghan C. Assessment of physiological signs associated with COVID-19 measured using wearable devices. NPJ Digit Med 2020;3:156.

66. Al Rajeh AM, Hurst JR. Monitoring of Physiological Parameters to Predict Exacerbations of Chronic Obstructive Pulmonary Disease (COPD): A Systematic Review. J Clin Med 2016;5:108.

67. Shah SA, Velardo C, Farmer A, et al. Exacerbations in Chronic Obstructive Pulmonary Disease: Identification and Prediction Using a Digital Health System. J Med Internet Res 2017;19:e69.

68. Orchard P, Agakova A, Pinnock H, et al. Improving Prediction of Risk of Hospital Admission in Chronic Obstructive Pulmonary Disease: Application of Machine Learning to Telemonitoring Data. J Med Internet Res 2018;20:e263.

69. Summers AM, Reed MJ. An evaluation of patient ownership and use and acceptability of smartphone technology within the emergency department. Eur J Emerg Med 2018;25:224-5. 
70. Slevin P, Kessie T, Cullen J, et al. Exploring the potential benefits of digital health technology for the management of COPD: a qualitative study of patient perceptions. ERJ Open Res 2019;5:00239-2018.

71. Fan KG, Mandel J, Agnihotri P, et al. Remote Patient Monitoring Technologies for Predicting Chronic Obstructive Pulmonary Disease Exacerbations: Review and Comparison. JMIR MHealth UHealth 2020;8:e16147.

72. Lehne M, Sass J, Essenwanger A, et al. Why digital medicine depends on interoperability. NPJ Digit Med 2019;2:79.

doi: 10.21037/jhmhp-20-119

Cite this article as: Carlin C, Taylor A, van Loon I, McDowell G, Burns S, McGinness P, Lowe DJ. Role for artificial intelligence in respiratory diseases-chronic obstructive pulmonary disease. J Hosp Manag Health Policy 202 1;5:27.
73. Panch T, Mattie H, Celi LA. The "inconvenient truth" about AI in healthcare. NPJ Digit Med 2019;2:77.

74. Ho TW, Huang CT, Chiu HC, et al. Effectiveness of Telemonitoring in Patients with Chronic Obstructive Pulmonary Disease in Taiwan-A Randomized Controlled Trial. Sci Rep 2016;6:23797.

75. RECEIVER: Digital Service Model for Chronic Obstructive Pulmonary Disease (COPD). ClinicalTrials. gov. [cited 2020 Aug 24]. Available online: https:// clinicaltrials.gov/ct2/show/NCT04240353 